
April 1941

\title{
AN APPARATUS FOR THE ABSORPTION OR GRAVIMETRIC DETERMINATION OF CONSTITUENTS OF A GAS MIX- TURE
}

\author{
By Martin Shepherd and Harry W. Bailey
}

ABSTRACT

The apparatus here described eliminates many of the undesirable features of certain types of "absorption trains" which are intended to remove constituents from gas mixtures or to determine them gravimetrically. It is adaptable to many uses, since auxiliary units, such as combustion apparatus, may be connected by means of interchangeable joints.

One of the commonest procedures associated with gas chemistry is the removal of one or more constituents of a gas mixture by reaction with solid or liquid reagents, for purification or for the gravimetric determination of absorbed constituents. The various types of apparatus used to conduct such reactions have been referred to as absorption or purification trains, or combustion trains (as employed in the analysis of steels, rocks, organic compounds, etc.). These so-called "trains" are usually constructed to meet the requirements of a particular experiment, and more often than not have a makeshift appearance. Even when they are assembled for more permanent use, little attention is given to convenience, and awkwardness has gradually become a characteristic of such apparatus.

When gases or vapors are to be absorbed and the amount of absorbed material is to be determined by increase in weight, the apparatus should have the following desirable features:

(1) The absorbers should be connected by means of well-ground joints, and not by rubber connections that will admit significant amounts of water or permit the loss of such gases as carbon dioxide.

(2) The connections should be made in such a way that the absorbers can be removed and replaced without disassembling the whole unit.

(3) The connections should be arranged so that other units, such as combustion tubes, can be inserted to increase the usefulness of the apparatus.

(4) The absorbers themselves should be as light as possible, so that their weight is not large in proportion to the weight of the absorbing reagent they contain.

(5) The absorbers should be easy to mount on the balance pan.

(6) The shape and finish of the outside surface of the absorbers should be such that they may be easily cleaned, and particularly so with respect to lubricant applied to the connections. 
(7) The absorbers should be designed so that they may be readily emptied and cleaned, and easily charged with reagent without fouling the grinding.

(8) The absorbers should bring the gas into intimate contact with the reagents, and, if possible, be suitable for both solid and liquid reagents.

(9) The absorbers must be provided with a suitable means of opening and closing.

(10) The unit should be mobile, so that it can be taken-in one piece-from job to job.

The apparatus shown in figure 1 possesses all of these desirable features. A detailed drawing of the absorber is given in figure 2 .

The absorber is blown from light-wall tubing. While the size may be varied for special purposes, that shown is suitable for general analytical work, and weighs approximately $14 \mathrm{~g}$. There are two parts: a ground cap, 1, bearing the side arms 4-4; and the tube, 7,

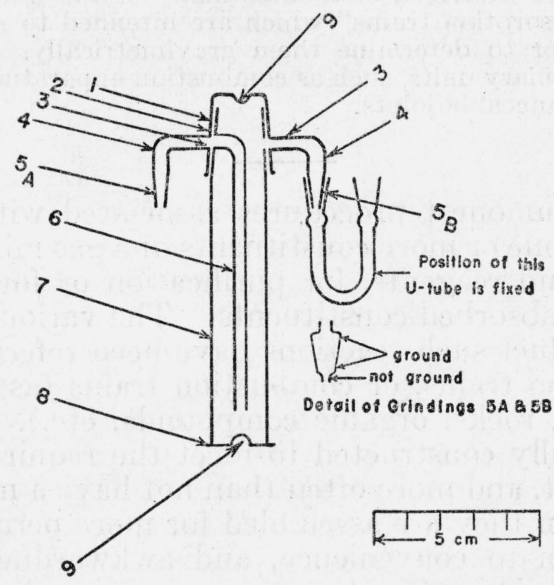

FIGURE 2.-Detail of absorber for gravimetric apparatus.

See text for explanation.

whose top is ground at 2 to correspond to the grinding of the cap. These grindings are standard interchangeable ones. The grindings at the end of the inlet and outlet arms, $5 A$ and $5 B$, are also interchangeable. The bores at $3-3$ are $4 \mathrm{~mm}$ in diameter. The inlet tube, 6 , brings the gas to the bottom of the absorber and permits use with solid or liquid reagents. The bottom of the absorber, 8 , is flattended so that it rests securely "on its own feet" on the balance pan or elsewhere. The cap and tube are dimpled at 9-9 to permit supporting in the manner to be described.

In charging the absorber, the reagent does not come into contact with the top grinding as is the case with the tubes generally used. The grindings $5 A$ and $5 B$ are male, and lubricant is accordingly easily dissolved from them before weighings are made. The lower portions of these grindings are cut in and left unground, so that lubricant does not tend to work into the tube-an old suggestion of E. W. Morley. The grindings, $5 A$ and $5 B$, are pointed vertically downward, so that they can be lifted directly out of corresponding female members. The female members are connected by a U-tube, which is permanently 


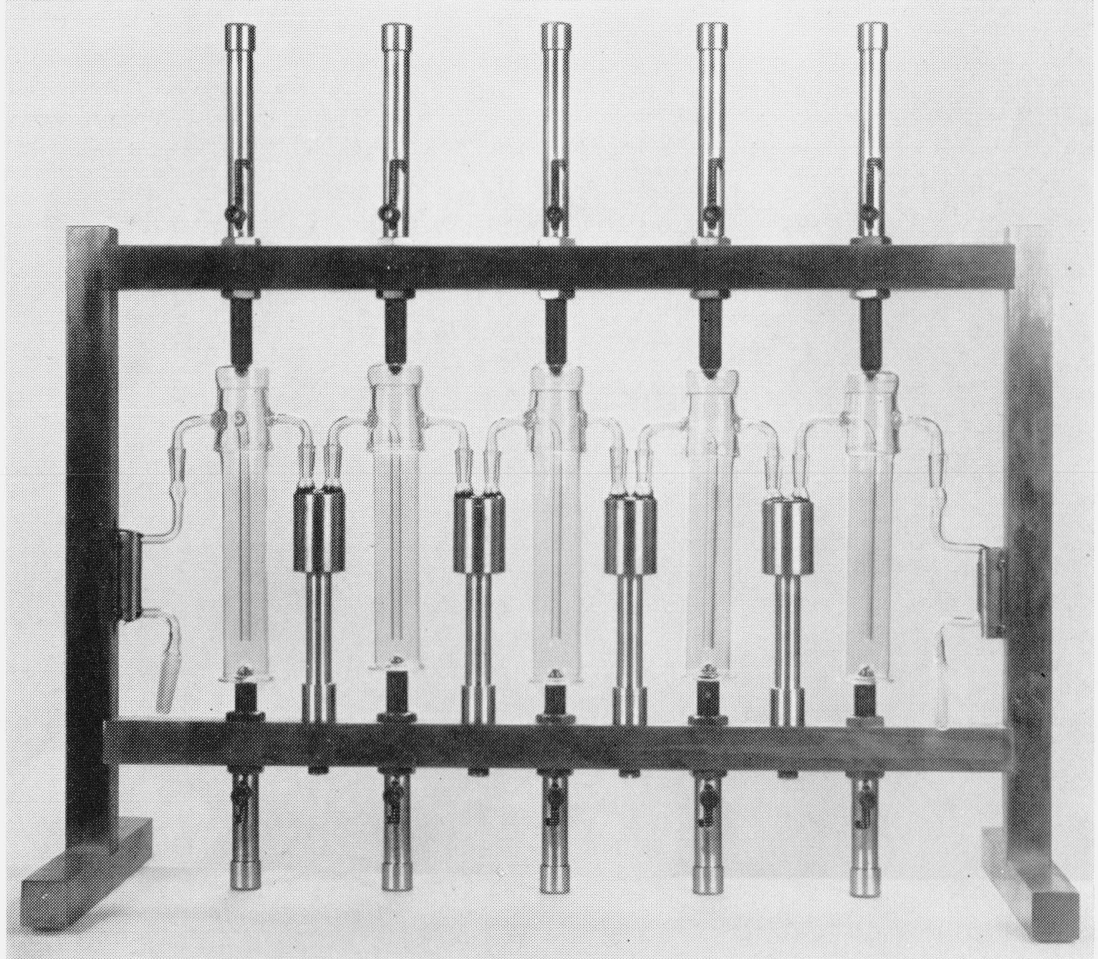

FiguRe 1.-Gravimetric apparatus. 
fixed in position. Thus, an absorber is removed from the train by merely lifting it out, and is replaced as easily. The generally employed method of disconnecting the absorbers by pulling apart horizontally-which means taking the whole apparatus apart-is excessively awkward. The absorbers are opened or closed by rotating the lower member, 7 , and not the cap or separate plugs as is the general practice.

Referring to the photograph (fig. 1), it will be seen that the U-tubes which terminate in the female grindings that provide the fixed connections to the absorbers, are mounted (in a cement) within the four brass cups and the two end clamps of the supporting frame. This mounting is done once for all by joining the whole train together with a low-melting thermoplastic, placing the whole in position on the frame, and pouring the cement to hold the U-connections permanently into the brass cups. Then the thermoplastic is dissolved from the grindings, and thereafter is replaced by lubricant.

It is necessary to hold the grindings of cap and tube (1 and '2), and of the side arms and U- connection ( $5 A$ and $5 B$ ) snugly together, since gas pressure within the train might force them apart. This is done by fiber plungers tapered at the end and bearing in the dimples 9-9 at top and bottom of the absorber. Compression is applied to these plungers by means of springs encased in the sets of five brass tubes at the top and bottom of the frame. The upper set of springs applies slightly more tension than the lower set. Thus the two types of grindings are held seated. The plungers are raised and lowered by small handles which may be locked in position to hold them away from the absorbers.

Since all grindings are interchangeable, convenient connection to other apparatus, gas supply, metering devices, etc., is available. In addition, one or more absorbers may be replaced by other units, such as combustion tubes. The apparatus can thus be adapted to many uses.

Washington, November 14, 1940. 